\title{
Hierarchical Bayesian auto-regressive models for large space time data with applications to ozone concentration modelling
}

\author{
Sujit Kumar Sahu* \\ School of Mathematics, University of Southampton, \\ Southampton, SO17 1BJ, UK. \\ Khandoker Shuvo Bakar \\ CSIRO Mathematics, Information and Statistics, \\ GPO Box 664, Canberra, ACT 2601, Australia.
}

Increasingly large volumes of space-time data are collected everywhere by mobile computing applications, and in many of these cases temporal data are obtained by registering events, for example telecommunication or web traffic data. Having both the spatial and temporal dimensions adds substantial complexity to data analysis and inference tasks. The computational complexity increases rapidly for fitting Bayesian hierarchical models, as such a task involves repeated inversion of large matrices. The primary focus of this paper is on developing space-time auto-regressive models under the hierarchical Bayesian setup. To handle large data sets, a recently developed Gaussian predictive process approximation method (Banerjee et al. [1]) is extended to include auto-regressive terms of latent space-time processes. Specifically, a spacetime auto-regressive process, supported on a set of a smaller number of knot locations, is spatially interpolated to approximate the original space-time process. The resulting model is specified within a hierarchical Bayesian framework and Markov chain Monte Carlo techniques are used to make inference. The proposed model is applied for analysing the daily maximum 8-hour average ground level ozone concentration data from 1997 to 2006 from a large study region in the eastern United States. The developed methods allow accurate spatial prediction of a temporally aggregated ozone summary, known as the primary ozone standard, along with its uncertainty, at any unmonitored location during the study period. Trends in spatial patterns of many features of the posterior predictive distribution of the primary standard, such as the probability of non-compliance with respect to the standard, are obtained and illustrated.

Keywords: Bayesian inference, big-n problem, Gaussian predictive process, ozone concentration, space-time modelling.

${ }^{*}$ Corresponding Author. Email: S.K.Sahu@soton.ac.uk 


\section{Introduction}

Many organisations collect spatial and spatio-temporal data routinely and on a regular basis for a variety of reasons. It has been estimated that up to $80 \%$ of all data stored in corporate databases may have a spatial component [2]. To gather intelligence from such large space-time data and to fully exploit the space-time dependencies for making predictive inference, there is an urgent need to develop fast and efficient statistical methods capable of handling large data sets. Spatio-temporal models, specified at the highest spatial and temporal resolutions, provide the capability for making inference at any aggregate spatial and/or temporal levels. For example, a model specified for the daily data can easily be aggregated to make inference on monthly or annual time scales and can also allow one to estimate the associated uncertainties in a coherent manner. Similarly, data modelled on a high resolution spatial scale can easily shed inferential light on any spatially aggregated quantity.

Incorporating both spatial and temporal correlations into the Bayesian hierarchical models is a challenging task, but this is the one that is receiving increasing interests from modellers from many fields of research and applications, see e.g., the recent book by Cressie and Wikle [3] and the references therein. Hierarchical dynamical spatio-temporal models are often specified to achieve the various modelling objectives. This paper will focus on the hierarchical auto-regressive models, see e.g., Sahu et al. [4] as a simpler sub-class of the dynamical spatio-temporal models since these are often found to be the best models among a large class of models under a variety of model choice and predictive model validation criteria, see e.g., [5]. Sahu and Bakar [6] show that these models also enjoy superior theoretical predictive properties and they also fit and validate better in real life modelling situations.

The hierarchical auto-regressive models, however, are not suitable for analysing large data sets observed over vast study regions such as the eastern United States (US). The problem here lies in inverting high dimensional spatial covariance matrices repeatedly in iterative model fitting algorithms. This is known as the big-n problem in literature (see e.g., Banerjee et al. [7]). The early approaches to solve the big$\mathrm{n}$ problem are mostly $a d-h o c$ as some data are either un-used or repeatedly used, e.g., [8] uses local kriging, [9] proposes subsampling from a large spatial dimension by a moving window approach. The gradual methodological improvement comes from the use of different approximation techniques of kriging equations, see e.g., a space-filling approach with geo-additive models by [10], a moving average technique by [11], and techniques based on using basis functions by [12]. Approaches based on spectral analysis to approximate the likelihood function have also been proposed by many authors, see e.g., [13] and [14]. Hartman and Hössjer [15] propose another technique based on efficient computations using sparse matrix calculation algorithms by exploiting the Gaussian Markov random fields ([16]) approximations. Reich et al. [17] use a spatial quantile regression approach by considering a non-Gaussian process 
in their models. They are able to reduce the dimension of the data by hierarchical modelling of a smaller number of estimated quantile parameters from the original observations.

Multi-resolution spatial models, see e.g., [18], [19], [20] are also able to provide fast model fitting and can also capture non-stationarity in the data. However, these models can be problematic to capture possible heterogeneity effects across vast spatial regions. Modelling spatial covariance functions with a low rank matrix ([21]) and a moderate rank matrix ([22]), fixed rank kriging ([23], [24]) can also handle the large data sets but there are several problems associated with the choice of the basis functions and their interpretations. A simpler approach based on an approximation using the Gaussian predictive processes (GPP) for modelling multivariate spatial data has been proposed by Banerjee et al. ([1]) and later on modified by [45]. This method does not require sub-sampling of the data and neither does it require specification of complex spatial basis functions.

Motivated by the need to model large data sets, this paper extends the GPP approximation technique of [1] to include auto-regressive terms of the latent underlying space-time process. This auto-regressive process is defined at a set of a smaller number of knot locations within the study region and then spatial interpolation, i.e. kriging, is used to approximate the original space-time process. The model is fully specified within a hierarchical Bayesian setup and is implemented using Markov chain Monte Carlo (MCMC) techniques.

The paper illustrates the method by analysing a very large data set that recorded the daily maximum 8-hour average ground level ozone concentration from 1997 to 2006 from a large number of monitoring sites located in the eastern US. Results from this study include a spatially interpolated model based map, first of its kind to our knowledge, showing the probability of non-compliance with respect to the primary standard in each of the years 1999-2006. The paper also compares the predictive performance of the GPP based model and the fully specified AR model of [4] using a moderately large subset of the full data set where both models are feasible. There is a considerable literature on space-time modeling of ground level ozone see e.g., [25], [26], [27], [28], [29], [30], [31], [32], and [33]. However, none of these articles model daily ozone concentration data for ten years observed over our study region of the eastern US using space-time models.

The rest of this paper is organised as follows. Section 2 provides exploratory analysis of the ozone concentration data set that we model in this paper. The hierarchical space time auto-regressive models for the big-n problem are developed in Section 3. Section 4 provides many different sensitivity analyses of the proposed method and also compares its performance with the currently available auto-regressive models ([4]) fitted to a subset of the original large data set. Analysis of the full eastern US ozone concentration data set using our methods is performed in Section 5. Section 6 sketches the relevance of this research to business and industry. Section 7 provides 
a few summary remarks and an appendix contains the full conditional distributions needed for implementing the Gibbs sampler.

\section{Exploratory analysis}

We consider the daily maximum 8-hour average ozone concentration data obtained from 691 monitoring sites in our study region in the eastern US. These sites are made up of 646 urban and suburban monitoring sites known as the National Air Monitoring Stations/State and Local Air Monitoring Stations (NAMS/SLAMS) and 45 rural sites monitored by the Clean Air Status and Trends Network (CASTNET). Out of the total 691 sites we set aside data from 69 randomly chosen sites for model validation purposes and use the data from the remaining 622 sites to fit the models. To relate ozone concentration with meteorological data we also have collected data from 746 weather monitoring sites within the study region. Figure 1 provides a map of the locations of these modelling, validation and weather monitoring sites.

We analyse daily data for $T=153$ days in every year during May to September since this is the high ozone season in the US. We consider these data for the 10 year period from 1997 to 2006 that allows us to study trend in ozone concentration levels. Thus we have a total of 1, 057, 230 observations and among them approximately $10.44 \%$ are missing which we assume to be at random, although there are some annual variation in this percentage of missingness.

The daily ozone concentration levels range from 0.22 to 246.22 in parts per billion (ppb) with a mean of $50.41 \mathrm{ppb}$. Figure 2 provides a side by side boxplot of the daily measurements for the 691 sites for each of the 10 years. The plot shows a general decreasing trend from 1997 to 2001, a peak in the year 2002 and then a decreasing trend until 2004. Slightly higher levels are seen in the last two years 2005 and 2006. We model ozone on the square-root scale since it encourages symmetry and variance stabilisation (see e.g., [4] and references therein), although other transformations such as the logarithm is possible.

The main purpose of the modelling exercise here is to assess compliance with respect to the primary ozone standard that states that the 3-year rolling average of the annual 4th highest daily maximum 8-hour average ozone concentration levels should not exceed 85 ppb, see e.g., [4]. Figure 3 plots the 4 th highest maximum and their 3-year rolling averages with a superimposed horizontal line at 85. As expected, the plot of the rolling averages is smoother than the plot of the annual 4th highest maximum values. The plots show that many sites are compliant with respect to the standard, but many others are not. In addition, the plot of the 3-year rolling averages shows a very slow downward trend. Both the plots show the presence of a few outlier sites which are perhaps due to site specific issues in air pollution, for example due to natural disasters such as forest fires. 
We consider three meteorological variables: daily maximum temperature in degree centigrade, relative humidity and wind speed as covariates for modelling the ozone concentration levels, following [34] and [4] who found these three to be the most significant explanatory variables, see also [29]. These three variables, however, have been observed at the 746 meteorological sites which do not coincide with the ozone monitoring sites (see Figure 1). Moreover, we need to have the values of these explanatory variables at a number of unmonitored sites for prediction purposes. We use simple kriging independently for the three meteorological variables using the fields package in $\mathrm{R}$ ([35]). This method is known to under-estimate the uncertainty in the predictions, see e.g., [17]. To address these concerns [36] considers a multiple kriging method where a new predictive value for each variable is generated independently at each MCMC iteration from the underlying estimated model for kriging. In this paper, however, we only report the results based on a single kriged value for each meteorological variable. Sahu et al. [4] detail a method where the covariate values at each time point are imputed using a separable multivariate spatio-temporal model. However, their approach is not suitable for the big-n problem of the current paper as discussed in the introduction. In their modelling developments, [4] use differences in successive day's meteorology as the covariates instead of the observations themselves so that the auto-regressive model using previous day's ozone levels can be adjusted by increment in meteorological values. In our modelling developments below we are not required to specify the models using the meteorological increments since we specify the auto-regressive models for random effects.

\section{Models}

Let $Z_{l}\left(\mathbf{s}_{i}, t\right)$ denote the observed value of the response, possibly after a transformation, at location $\mathbf{s}_{i}, i=1, \ldots, n$ on day $t$ within year $l$ for $l=1, \ldots, r$ and $t=1, \ldots, T$. These two time indices can also be used to specify the hour within day or day within month and so on. Let $\mathbf{Z}_{l t}=\left(Z_{l}\left(\mathbf{s}_{1}, t\right), \ldots, Z_{l}\left(\mathbf{s}_{n}, t\right)\right)^{\prime}$. Further, suppose that $x_{l j}(\mathbf{s}, t)$ denotes the value of the $j$ th covariate, $j=1, \ldots, p$ on day $t$ in year $l$ and let

$$
X_{l t}=\left(\begin{array}{c}
\mathbf{x}_{l}^{\prime}\left(\mathbf{s}_{1}, t\right) \\
\vdots \\
\mathbf{x}_{l}^{\prime}\left(\mathbf{s}_{n}, t\right)
\end{array}\right) \text {. }
$$

With these general notations several versions of auto-regressive models are described next. 


\subsection{Auto-regressive models developed by Sahu et al. [4]}

We first write down the hierarchical auto-regressive models developed in [4]. The first stage of their modelling hierarchy assumes the measurement error model:

$$
\mathbf{Z}_{l t}=\mathbf{O}_{l t}+\boldsymbol{\epsilon}_{l t}, l=1, \ldots r, t=1, \ldots, T
$$

where $\boldsymbol{\epsilon}_{l t}=\left(\epsilon_{l}\left(\mathbf{s}_{1}, t\right), \cdots, \epsilon_{l}\left(\mathbf{s}_{n}, t\right)\right)^{\prime} \sim N\left(0, \sigma_{\epsilon}^{2} I\right)$. Here $\mathbf{O}_{l t}=\left(O_{l}\left(\mathbf{s}_{1}, t\right), \ldots, O\left(\mathbf{s}_{n}, t\right)\right)^{\prime}$ denotes the true value corresponding to $Z_{l}\left(\mathbf{s}_{i}, t\right)$ where

$$
\mathbf{O}_{l t}=\rho \mathbf{O}_{l t-1}+X_{l t} \boldsymbol{\beta}+\boldsymbol{\zeta}_{l t}, l=1, \ldots r, t=1, \ldots, T \text {. }
$$

where $\boldsymbol{\zeta}_{l t}=\left(\zeta_{l}\left(\mathbf{s}_{1}, t\right), \ldots, \zeta_{l}\left(\mathbf{s}_{n}, t\right)\right)^{\prime}$ and we assume that $\boldsymbol{\zeta}_{l t} \sim G P_{n}\left(0, \sigma^{2} \psi(d ; \phi)\right)$ where $G P_{n}\left(0, \sigma^{2} \psi(d ; \phi)\right)$ denotes the $n$-dimensional Gaussian process with mean 0 and covariance function given by $\sigma^{2} \psi(d ; \phi)$ where $\sigma^{2}$ and $\phi$ denote the common spatial variance and the decay parameter, respectively and $d$ denotes the distance. Here $\psi(d ; \phi)$ denotes the correlation function which we take to be the exponential correlation function $\psi(d ; \phi)=\exp (-d \phi)$ in our illustration, although other choices can be adopted. The initial condition is given by

$$
\mathrm{O}_{l 0}=\gamma_{l}+\mu_{l} \mathbf{1}, l=1, \ldots r
$$

where $\mathbf{1}$ is the vector of dimension $n$ with all elements unity and $\gamma_{l}=\left(\gamma_{l}\left(\mathbf{s}_{1}\right), \ldots, \gamma_{l}\left(\mathbf{s}_{n}\right)\right)^{\prime}$ is assumed to follow $G P_{n}\left(0, \sigma_{l}^{2} \psi\left(d ; \phi_{0}\right)\right)$ independently for each $l=1, \ldots, r$. Note that these initial random variables $O_{l}\left(\mathbf{s}_{i}, 0\right)$ are centred at $\mu_{l}$ and not at zero since $O_{l}\left(\mathbf{s}_{i}, 0\right)$ represent the true response. The model is completed by assuming $\mu_{l} \sim N\left(0,10^{4}\right)$ and proper inverse gamma prior distributions independently for each $\sigma_{l}^{2}$, see [4] for further details. Henceforth, we shall denote this version of the AR models as ARHIER.

This version of the AR models is not suitable for adaptation with the GPP approximation proposed by [1] since each $Z_{l}\left(\mathbf{s}_{i}, t\right)$ is paired with a true level $O_{l}\left(\mathbf{s}_{i}, t\right)$ that cannot be reduced in dimension. Next we develop a modified version of these models that will enable us to reduce the dimension of the random effects.

\subsection{Modified hierarchical auto-regressive models}

The ARHIER model assumes the AR model for the true values of the modelled response $\mathbf{O}_{l t}$. Following [37] we modify this model so that the modified version does not assume a true level $O_{l}\left(\mathbf{s}_{i}, t\right)$ for each $Z_{l}\left(\mathbf{s}_{i}, t\right)$ but instead assumes a space-time random-effect denoted by $\eta_{l}\left(\mathbf{s}_{i}, t\right)$. It then assumes an AR model for these space-time random effects.

The top level general space-time random effect model is assumed to be:

$$
\mathbf{Z}_{l t}=X_{l t} \boldsymbol{\beta}+\boldsymbol{\eta}_{l t}+\boldsymbol{\epsilon}_{l t}, l=1, \ldots r, t=1, \ldots, T
$$


where $\boldsymbol{\epsilon}_{l t} \sim N\left(0, \sigma_{\epsilon}^{2} I\right)$ where $I$ is the identity matrix. In the next stage of the modelling hierarchy the AR model is assumed as:

$$
\boldsymbol{\eta}_{l t}=\rho \boldsymbol{\eta}_{l t-1}+\boldsymbol{\delta}_{l t}
$$

where $\boldsymbol{\delta}_{l t} \sim G P_{n}\left(0, \sigma^{2} \psi(d ; \phi)\right)$. The initial condition is assumed to be:

$$
\boldsymbol{\eta}_{l}\left(\mathbf{s}_{i}, 0\right) \sim G P_{n}\left(0, \sigma_{0}^{2} \psi\left(d ; \phi_{0}\right)\right)
$$

where $\sigma_{0}^{2}$ and $\phi_{0}$ are unknown parameters.

Note that the marginal mean of the random effects $\boldsymbol{\eta}_{l t}$ is zero, but the conditional mean given $\boldsymbol{\eta}_{l t-1}$ is no longer zero due to the auto-regressive specification (5). This specification also implies a non-stationary marginal covariance function for $\boldsymbol{\eta}_{l t}$ that does not need to be explicitly derived nor is it required since model fitting proceeds through the conditional specification (5). Lastly, we note that higher order autoregressive terms can be included in (5). However, those are not considered here since their inclusion will only weaken both the spatial and lag-1 temporal correlations without providing significant additional descriptive ability.

\subsection{Auto-regressive models with GPP approximations}

The auto-regressive models specified in Section 3.2 create a random effect $\eta_{l}\left(\mathbf{s}_{i}, t\right)$ in (4) corresponding to each data point $Z_{l}\left(\mathbf{s}_{i}, t\right)$. This will lead to the big-n problem, as discussed in the Introduction when $n$ is large. To overcome this problem we propose a dimension reduction technique through a kriging approximation following [1] as follows. The main idea here is to define the random effects $\eta_{l}\left(\mathbf{s}_{i}, t\right)$ at a smaller number of locations, called the knots, and then use kriging to predict those random effects at the data locations. The auto-regressive model is only assumed for the random effects at the knot locations and not for all the random effects at the observation location. The method proceeds as follows.

At the top level we continue to assume the model (4), but we do not specify $\boldsymbol{\eta}_{l t}$ directly through the auto-regressive model (5). Instead, we select $m<<n$ knot locations, denoted by by $\mathbf{s}_{1}^{*}, \ldots, \mathbf{s}_{m}^{*}$ within the study region and let the spatial random effects at these locations at time $l$ and $t$ be denoted by $\mathbf{w}_{l t}=\left(w_{l}\left(\mathbf{s}_{1}^{*}, t\right), \ldots, w_{l}\left(\mathbf{s}_{m}^{*}, t\right)\right)^{\prime}$. Discussion regarding the choice of these locations is given in Section 4.1. Assuming an underlying Gaussian process independently at each time point $l$ and $t$, [1] show that the process $\boldsymbol{\eta}_{l t}$ can be approximated by

$$
\tilde{\boldsymbol{\eta}}_{l t}=A \mathbf{w}_{l t}
$$

with $A=C S_{w}^{-1}$ where $C$ denotes the $n$ by $m$ cross-correlation matrix between $\boldsymbol{\eta}_{l t}$ and $\mathbf{w}_{l t}$, and $S_{w}$ is the correlation matrix of $w_{l t}$. Note that the common spatial variance parameter does not affect the above since it cancels in the product $C S_{w}^{-1}$. Also, there 
is no contribution of the means of either $\boldsymbol{\eta}_{l t}$ or $\mathbf{w}_{l t}$ in the above since those means are assumed to be 0 .

The proposal here is to use the GPP approximation $\tilde{\boldsymbol{\eta}}_{l t}$ instead of $\boldsymbol{\eta}_{l t}$ in the top level model (4), thus we assume that:

$$
\mathbf{Z}_{l t}=X_{l t} \boldsymbol{\beta}+\tilde{\boldsymbol{\eta}}_{l t}+\boldsymbol{\epsilon}_{l t}, l=1, \ldots r, t=1, \ldots, T
$$

where $\tilde{\boldsymbol{\eta}}_{l t}$ is as given in (7). Analogous to (5), we specify $\mathbf{w}_{l t}$ at the knots conditionally, given $\mathbf{w}_{l t-1}$, by

$$
\mathbf{w}_{l t}=\rho \mathbf{w}_{l t-1}+\xi_{l t},
$$

where $\boldsymbol{\xi}_{l t} \sim G P_{m}\left(0, \sigma_{w}^{2} \psi(d ; \phi)\right)$ independently. Again we assume that $\mathbf{w}_{l 0} \sim N\left(\mathbf{0}, \sigma_{l}^{2} S_{0}\right)$ independently for each $l=1, \ldots, r$, where the elements of the covariance matrix $S_{0}$ are obtained using the correlation function, $\psi\left(d ; \phi_{0}\right)$, i.e. the same correlation function as previously but with a different variance component for each year and also possibly with a different decay parameter $\phi_{0}$ in the correlation function. Henceforth, we shall call this model ARGPP.

A few remarks are in order. The above modelling specifications are justified using the usual hierarchical modelling philosophies in the sense that the top level model is a mixed model with mean zero random effects and these random effects have structured correlations as implied by the spatial auto-regressive model at the second stage (9). These two model equations, together with the initial condition, however, are neither intended to, nor will ever exactly imply the auto-regressive model (5) for the original random effects $\boldsymbol{\eta}_{l t}$ except for trivial cases such as the one where $m=n$ and all the knot locations coincide with the data locations. In general such a property can never be expected to hold without further conditions. However, the full dimensional autoregressive model (5), if feasible, will provide a better fit than the proposed model based on the GPP approximation. We also note that it is straightforward to work with an alternative independent model for $\mathbf{w}_{l t}$ instead of the auto-regressive model (9). Such a model, however, does not keep any explicit provision for modelling temporal dependence and, as a result will not be appropriate in many practical situations, and is not considered any further in the paper.

\subsubsection{Joint posterior details}

Define $N=n r T$ and let $\boldsymbol{\theta}$ denote all the parameters $\boldsymbol{\beta}, \rho, \sigma_{\epsilon}^{2}, \sigma_{w}^{2}, \phi, \phi_{0}, \sigma_{l}^{2}, l=1, \ldots, r$. Further, let $\mathbf{z}^{*}$ denote the missing data and $\mathbf{z}$ denote all the non-missing data. The log of the joint posterior distribution for the models in equations (8) and (9), denoted 
by $\log \pi\left(\boldsymbol{\theta}, \mathbf{z}^{*} \mid \mathbf{z}\right)$ is written as:

$$
\begin{aligned}
& -\frac{N}{2} \log \left(\sigma_{\epsilon}^{2}\right)-\frac{1}{2 \sigma_{\epsilon}^{2}} \sum_{l=1}^{r} \sum_{t=1}^{T}\left(\mathbf{Z}_{l t}-\mathbf{X}_{l t} \boldsymbol{\beta}-A \mathbf{w}_{l t}\right)^{\prime}\left(\mathbf{Z}_{l t}-\mathbf{X}_{l t} \boldsymbol{\beta}-A \mathbf{w}_{l t}\right) \\
& -\frac{m r T}{2} \log \left(\sigma_{w}^{2}\right)-\frac{r T}{2} \log \left(\left|S_{w}\right|\right)-\frac{1}{2 \sigma_{w}^{2}} \sum_{l=1}^{r} \sum_{t=1}^{T}\left(\mathbf{w}_{l t}-\rho \mathbf{w}_{l t-1}\right)^{\prime} \mathbf{S}_{w}^{-1}\left(\mathbf{w}_{l t}-\rho \mathbf{w}_{l t-1}\right) \\
& -\frac{m}{2} \sum_{l=1}^{r} \log \left(\sigma_{l}^{2}\right)-\frac{r}{2} \log \left(\left|S_{0}\right|\right)-\frac{1}{2} \sum_{l=1}^{r} \frac{1}{\sigma_{l}^{2}} \mathbf{w}_{l 0} \mathbf{S}_{0}^{-1} \mathbf{w}_{l 0}+\log \pi(\boldsymbol{\theta})
\end{aligned}
$$

where, $\log \pi(\boldsymbol{\theta})$ is the $\log$ of the prior distribution for the parameter $\boldsymbol{\theta}$. We assume the prior distributions $\boldsymbol{\beta} \sim N\left(\mathbf{0}, 10^{4}\right), \rho \sim N\left(0,10^{4}\right) I(0<\rho<1)$. Further, the prior distributions for the variance parameters are: $1 / \sigma_{\epsilon}^{2} \sim G(a, b), 1 / \sigma_{w}^{2} \sim G(a, b)$, where the Gamma distribution has mean $a / b$. We shall choose the values of $a$ and $b$ in such a way that guarantees a proper prior distribution for these variance components, see Section 4 below.

\subsubsection{Prediction details}

According to the top-level model (8), the response $Z_{l}\left(\mathbf{s}^{\prime}, t\right)$ at a new site $\mathbf{s}^{\prime}$ and time $l$ and $t$ is given by

$$
Z_{l}\left(\mathbf{s}^{\prime}, t\right)=\mathbf{x}_{l}\left(\mathbf{s}^{\prime}, t\right)^{\prime} \boldsymbol{\beta}+\tilde{\eta}_{l}\left(\mathbf{s}^{\prime}, t\right)+\epsilon_{l}\left(\mathbf{s}^{\prime}, t\right), l=1, \ldots r, t=1, \ldots, T,
$$

where $\mathbf{x}_{l}\left(\mathbf{s}^{\prime}, t\right)$ denotes the covariate value at the new location at time $l$ and $t$, and the scalar $\tilde{\eta}_{l}\left(\mathbf{s}^{\prime}, t\right)$ is obtained using the following equation, obtained analogously as $(7)$,

$$
\tilde{\eta}_{l}\left(\mathbf{s}^{\prime}, t\right)=\mathbf{c}^{\prime}\left(\mathbf{s}^{\prime}\right) S_{w}^{-1} \mathbf{w}_{l t}
$$

where the $k$ th element of the $m$ by 1 vector $\mathbf{c}\left(\mathbf{s}^{\prime}\right)$ is given by $\psi(d ; \phi)$ where $d$ is the distance between the sites $\mathbf{s}_{k}^{*}$ and $\mathbf{s}^{\prime}$.

Prediction is straightforward under any MCMC sampling scheme. At each iteration, $j$ say, first, one obtains the approximation $\tilde{\eta}_{l}^{(j)}\left(\mathbf{s}^{\prime}, t\right)$ calculated using the current parameter iterates $\boldsymbol{\theta}^{(j)}$ and $\mathbf{w}_{l t}^{(j)}$. The next step is to generate a new $Z_{l}^{(j)}\left(\mathbf{s}^{\prime}, t\right)$ using the model (11) and plugging in the current iterates $\boldsymbol{\theta}^{(j)}$ and $\mathbf{w}_{l t}^{(j)}$. These MCMC iterates are transformed to the original scale by using the inverse of the transformation used to model the original response. Let $q(\cdot)$ denote this inverse transformation. For example, if observations have been modelled on the square root scale then $q(z)=z^{2}$, so that we obtain the predictions on the original scale. The MCMC iterates on the original scale are summarised as the prediction value at the end of the MCMC run, and the uncertainty in this prediction value is also simultaneously estimated. This ability to estimate the uncertainty of the predictions on the original scale is an essential attractive feature of the MCMC methods since no approximation or analytical calculation or bootstrapping is required. 


\subsubsection{Predicting the annual summaries}

Aggregated temporal summaries are also simulated at each MCMC iteration using the prediction iterate on the original scale, see e.g., [4]. For example, for each year $l$ we obtain the annual $4 t h$ highest maximum ozone value by calculating the $4 t h$ highest value, denoted by $f_{l}^{(j)}\left(\mathbf{s}^{\prime}\right)$, of the transformed $q\left(Z_{l}^{(j)}\left(\mathbf{s}^{\prime}, t\right)\right), t=1, \ldots, T$. The 3-year rolling averages of these are given by

$$
g_{l}^{(j)}\left(\mathbf{s}^{\prime}\right)=\frac{f_{l-2}^{(j)}\left(\mathbf{s}^{\prime}\right)+f_{l-1}^{(j)}\left(\mathbf{s}^{\prime}\right)+f_{l}^{(j)}\left(\mathbf{s}^{\prime}\right)}{3}, l=3, \ldots, r .
$$

Following [4], we can also obtain covariate (meteorologically) adjusted trend values by considering the random effects, $\tilde{\eta}_{l}^{(j)}\left(\mathbf{s}^{\prime}, t\right)$ since these are the residuals after fitting the regression model $\mathbf{x}_{l}\left(\mathbf{s}^{\prime}, t\right)^{\prime} \boldsymbol{\beta}^{(j)}$. These realizations are first transformed to the original scale by obtaining $q\left(\tilde{\eta}_{l}^{(j)}\left(\mathbf{s}^{\prime}, t\right)\right)$. These transformed values are then averaged over a year to obtain the covariate adjusted value, denoted by $h_{l}\left(\mathbf{s}^{\prime}\right)$, for that year, i.e.

$$
h_{l}^{(j)}\left(\mathbf{s}^{\prime}\right)=\frac{1}{T} \sum_{t=1}^{T} q\left(\tilde{\eta}_{l}^{(j)}\left(\mathbf{s}^{\prime}, t\right)\right)
$$

A further measure of trend called the relative percentage trend (RPCT) for any year $l$ relative to the year $l^{\prime}$ is defined by

$$
100 \times\left(h_{l}^{(j)}\left(\mathbf{s}^{\prime}\right)-h_{l^{\prime}}^{(j)}\left(\mathbf{s}^{\prime}\right)\right) / h_{l^{\prime}}^{(j)}\left(\mathbf{s}^{\prime}\right)
$$

is also simulated at each MCMC iteration $j$. The MCMC iterates $f_{l}^{(j)}\left(\mathbf{s}^{\prime}\right), g_{l}^{(j)}\left(\mathbf{s}^{\prime}\right)$, $h_{l}^{(j)}\left(\mathbf{s}^{\prime}\right)$ and also any relative trend values are summarised at the end of the MCMC run to obtain the corresponding estimates along with their uncertainties.

\subsubsection{Forecasting}

Forecasting is also straightforward under the MCMC methods. To obtain the one-step forecasts at any location $\mathbf{s}^{\prime}$ we note that:

$$
Z_{l}\left(\mathbf{s}^{\prime}, T+1\right)=\mathbf{x}_{l}\left(\mathbf{s}^{\prime}, T+1\right)^{\prime} \boldsymbol{\beta}+\tilde{\eta}_{l}\left(\mathbf{s}^{\prime}, T+1\right)+\epsilon_{l}\left(\mathbf{s}^{\prime}, T+1\right), l=1, \ldots r,
$$

where $\tilde{\eta}_{l}\left(\mathbf{s}^{\prime}, T+1\right)$ is to be obtained using (12). This requires $\mathbf{w}_{l T+1}$ which we obtain using (9). Thus at each MCMC iteration we draw a forecast iterate $Z_{l}^{(j)}\left(\mathbf{s}^{\prime}, T+1\right)$ from the normal distribution with mean $\mathbf{x}_{l}\left(\mathbf{s}^{\prime}, T+1\right)^{\prime} \boldsymbol{\beta}^{(j)}+\tilde{\eta}_{l}^{(j)}\left(\mathbf{s}^{\prime}, T+1\right)$ and variance $\sigma_{\epsilon}^{2(j)}$. Similar forecasting using the Sahu et al. [4] method is more computationally involved since for each new $\mathbf{s}^{\prime}$ that requires simulation $O_{l}\left(\mathbf{s}^{\prime}, t\right)$ for all $t=0, \ldots, T+1$. Forecasting is no longer pursued in this paper, but is considered in a companion paper [38]. 


\subsection{Software for computation}

The methods developed above require careful programming as no suitable software package is available for Bayesian hierarchical modelling of space-time data. The $\mathrm{R}$ package spBayes developed by Finley et al. [39] can fit multivariate spatial models but at the moment is not able to fit rich temporal data sets such as the ones introduced in Section 2. We have developed the $\mathrm{R}$ package spTimer, see [40], in a companion paper that will be available from the $\mathrm{R}$ archive network CRAN (http://cran.rproject.org/).

\section{Results for a smaller data set}

This section studies the sensitivity of the proposed model ARGPP and its performance against the Sahu et al. model ARHIER using data from a smaller subset of four states Illinois, Indiana, Ohio, and Kentucky. There is a total of 164 ozone monitoring sites inside these four states that allows us to study the various issues in much detail without having to undertake the large computational burden required to analyse the full data set. Data from 16 randomly chosen sites are set aside for validation purposes, although this choice is repeated 7 times in an experiment to see the effect of the choice of these validation sites. The validation statistics, defined below, are based on daily observations for 153 days in each of 10 years from the 16 hold-out sites. Out of these 24480 observations, $3472(14 \%)$ are missing and have been excluded from calculating the validation statistics. Figure 4 provides a map of the four states showing the locations of the 164 ozone monitoring sites and the 88 meteorological sites and also the locations of the 107 knot points for illustration.

Sensitivity studies are performed using two model validation criteria: the root mean square error (RMSE) and the mean absolute error (MAE) summarising the closeness of the out of sample predictions to observed values. The RMSE criterion is the square root of the validation mean square error criterion given by:

$$
\mathrm{VMSE}=\frac{1}{k r T} \sum_{j=1}^{k} \sum_{l=1}^{r} \sum_{t=1}^{T}\left(\hat{Z}_{l}\left(\mathbf{s}_{j}, t\right)-z_{l}\left(\mathbf{s}_{j}, t\right)\right)^{2}
$$

where $\hat{Z}_{l}\left(\mathbf{s}_{j}, t\right)$ is the model predicted value of $Z_{l}\left(\mathbf{s}_{j}, t\right)$ at year $l$ and time $t$ at the validation site $j$, and $k$ is the number of validation sites. In the calculations for VMSE, the terms corresponding to the missing observations must be omitted; in such a case the divisor must be adjusted appropriately as well. The MAE criterion is similarly defined where the squares of the validation errors in (13) are replaced by the absolute errors.

For model comparison purposes we use the predictive model choice criterion (PMCC), see e.g., [41], that is suitable for comparing models with normally dis- 
tributed error distributions and is given by:

$$
\mathrm{PMCC}=\sum_{i=1}^{n} \sum_{l=1}^{r} \sum_{t=1}^{T} E\left(Z_{l}\left(\mathbf{s}_{i}, t\right)_{\mathrm{rep}}-z_{l}\left(\mathbf{s}_{i}, t\right)\right)^{2}+\sum_{i=1}^{n} \operatorname{Var}\left(Z_{l}\left(\mathbf{s}_{i}, t\right)_{\mathrm{rep}}\right),
$$

where $Z_{l}\left(\mathbf{s}_{i}, t\right)_{\text {rep }}$ denotes a future replicate of the data $Z_{l}\left(\mathbf{s}_{i}, t\right)$. The first term in the above is a goodness of fit term $(G)$ while the second is a penalty term $(P)$ for model complexity. The model with the smallest value of PMCC is selected among the competing models. Thus, to be selected a model must strike a good balance between goodness of fit and model complexity. The terms $P$ and $G$ are estimated using composition sampling, at each MCMC iteration $k$ we first draw parameter

values from the posterior distribution and then $Z_{l}\left(\mathbf{s}_{i}, t\right)_{\text {rep }}^{(k)}$ from the model equations conditional on the drawn parameter values.

In all our illustrations below we have diagnosed MCMC convergence by visual examination of the time-series plots of the MCMC iterates. We have also used multiple parallel runs and calculated the Gelman and Rubin statistic ([42]) that was satisfactory in every case. We have used 5000 iterations to make inference after discarding first 1000 iterates to mitigate the effect of initial values.

\subsection{Sensitivity study}

\section{Number of knots}

To study the sensitivity of the out of sample predictions with respect to the number of knots, we define five different sets of regular grid locations: $6 \times 6,8 \times 8,10 \times 10$, $12 \times 12$ and $14 \times 14$ over our study region of the four states. From these regular grids, we only keep the points that fall inside the boundary of the four states. This gives us the knots with 26, 40, 60, 107 and 138 locations respectively. Figure 4 illustrates the case with with 107 knot locations.

Both the validation criteria: RMSE and MAE, provided in Table 1, show that the ARGPP model is able to predict better for larger knot sizes. This is expected since model fitting gets better with larger knot sizes, as noted previously in Section 3. However, Table 1 also shows that the gains in the validation criteria are not substantial beyond the knot size of 107. Henceforth, we shall adopt the set of knots with 107 locations to illustrate the results in this section.

\section{Prior distributions}

Bayesian model fitting and predictions are often influenced by the adopted prior distribution. In our modelling efforts, we have always used vague prior distributions for the mean parameters, hence our results are not sensitive to that choice. However, we have used the proper gamma prior distribution with hyper-parameters $a$ and $b$ for the two variance components $\sigma_{\epsilon}^{2}$ and $\sigma_{w}^{2}$. We have used three pairs of values of $a$ and $b$ : $(2,1),(1,1),(10,10)$ that gave us RMSE values 6.07, 6.10 and 6.10 respectively. The corresponding MAE values were 4.37, 4.41 and 4.41 respectively. 
Hence, the predictions are not very sensitive to the choice of hyperparameter values and henceforth we adopt the pair of values 2 and 1 for $a$ and $b$.

\section{Sampling method for $\phi$}

The choice of the estimation method for the spatial decay parameter, $\phi$, has an effect on the prediction results. In a classical inference setting it is not possible to consistently estimate both $\phi$ and $\sigma_{w}^{2}$ in a typical model for spatial data with a covariance function belonging to the Matèrn family, see [43]. Moreover, [13] shows that spatial interpolation is sensitive to the product $\sigma_{w}^{2} \phi$ but not to either one individually. That is why, [44] choose the decay parameter using an empirical Bayes (EB) approach by minimising the RMSE and estimate the variance parameter $\sigma_{w}^{2}$ conditionally on the fixed estimated value of $\phi$. Here we avoid the EB approach by sampling $\phi$ from its full conditional distribution in two different ways corresponding to two different prior distributions. The first of these is a discrete uniform prior distribution on 20 equally spaced mass points in the interval $(0.001,0.1)$. Here, although time consuming, the full conditional distribution is discrete and sampling is relatively straightforward, see the appendix. The second strategy, corresponding to a continuous uniform prior distribution in the interval $(0.001,0.1)$ for $\phi$, is the random walk Metropolis-Hastings algorithm tuned to have an optimal acceptance rate between $20 \%$ to $40 \%$, see the appendix. In one of our implementations, we have been able to tune this algorithm to achieve an acceptance rate of $32.4 \%$ that gave an RMSE value of 6.07 . The RMSE value for the discrete sampling method was 6.16. The respective MAE values were 4.37 and 4.46. Thus the Metropolis-Hastings method provides better validation results and henceforth this method is adopted.

\section{Choice of hold-out data}

Results may be sensitive to the particular hold-out data set. We study this as follows. We randomly choose 10 different hold-out data sets, each of them consisting of observations from 16 out of the 164 total monitoring sites in the study region of four states. The RMSEs for these 7 hold-out data sets vary between 6.07 and 6.20 whereas the MAEs are between 4.36 and 4.55. This shows that the validation results are not much sensitive to the choice of the hold-out data. Henceforth, we adopt the original hold out data set that had the RMSE and MAE values 6.07 and 4.37, respectively.

\subsection{Comparison with the ARHIER model}

This section is devoted to comparing the proposed ARGPP models with the ARHIER models. Note that the ARGPP does not directly approximate ARHIER and hence the latter is not likely to be uniformly better than the former, and hence this comparison is meaningful. Both the models use the same three covariates, namely, maximum temperature (MaxTemp), relative humidity $(\mathrm{RH})$ and wind speed (WDSP). In both the cases we also adopt the same prior distributions and use the Metropolis-Hastings 
sampling algorithm for sampling the spatial decay parameters. In the GPP based proposed model we use 107 knot points as decided above.

The estimates of the parameters of the two models are provided in Table 2, except for the parameters $\mu_{l}$ and $\sigma_{l}^{2}$ under the ARHIER model and $\sigma_{l}^{2}$ under the ARGPP model, $l=1, \ldots, r$ since those estimates are not interesting for model comparison purposes. Both the models show significant effect of the three covariates, although the effects get attenuated under the ARHIER model due to the presence of the temporal auto-regression. Further discussion about these effects is provided in Section 5. However, there are large differences between the two models as regards to the estimates of spatial and temporal correlations. The temporal correlation under the ARHIER (0.523) is much larger than the same for the ARGPP model (0.092). This is due to the fact that the auto-regressive model for the Sahu et al. version is assumed for the true ozone levels which are highly temporally correlated, whereas the GPP based model assumes the auto-regression for the latent random effects which are also significantly temporally correlated but at a magnitude lower than that for the true ozone levels in ARHIER. However, to compensate for this low value of temporal correlation, the GPP based model has estimated a much higher level of spatial correlation since the spatial decay of 0.0036 is much smaller for this model compared to the same, 0.012, for the full hierarchical AR model. The estimates of the variance components, under both the models, show that more variation is explained by the spatial effects than the pure error.

The two models are compared using the PMCC and the two model validation criteria: RMSE and MAE. We also report the nominal coverage of the $95 \%$ prediction intervals for the out of sample validation data. These three validation statistics are based on 21,008 (=24480-3472) daily observations as noted above. Model comparison results presented in Table 3 almost uniformly give evidence in favour of the the proposed GPP based models. Components of the PMCC show that the GPP based model provides a much better fit than the ARHIER. Both the RMSE and MAE are also better for the proposed model. However, the nominal coverage is slightly smaller for the proposed method, but this is not much of a cause for concern since both are close to 95\%. The ARGPP model fitting requires about 2.24 hours of computing time while the full ARHIER model takes about 7.86 hours. Thus the ARGPP implementation requires less than a third of the computing time needed for fitting the ARHIER model. In conclusion, the ARGPP model not only provides a faster and better fit but also validates better than the ARHIER. In the next section, for the full eastern US data, we shall only consider the GPP based model. 


\section{Analysis of the full eastern US data set}

We now analyse the full eastern US data set introduced in Section 2. We use data from 622 monitoring sites to model and the data for the remaining 69 sites are set aside for validation, see Figure 1. We continue to use the three meteorological variables as covariates in the model. We choose the same prior and the Metropolis-Hastings sampling method for the spatial decay parameter $\phi$. To select the number of knots we start with regular grid sizes of $12 \times 12,15 \times 15,20 \times 20$ and $25 \times 25$ and then only retain the points inside the land boundary of the eastern US that gives us 68 , 105, 156 and 269 points respectively. As in previous section we fit and predict using the model with these knot sizes and obtain the two validation statistics: RMSE and MAE in Table 4. As has already been seen in Section 4, the performance gets better with increasing grid sizes, but the improvement in performance is only marginal when the grid size goes up to 269 from 156. The much smaller computational burden with 156 knot points outweighs this marginal improvement in the validation statistics. Henceforth we proceed with the grid size 156 in our analysis. Figure 5 provides a map of the eastern US with these grid points superimposed.

Parameter estimates of the fitted model with 156 knot points are provided in Table 5. All three covariates, MaxTemp, RH and WDSP, remain significant in the spatio-temporal model with a positive effect of MaxTemp and negative effects of the other two. This is in accordance with the results in the literature in ozone modelling, see e.g., [4]. The auto-regressive parameter is also significant and the pure error variance $\sigma_{\epsilon}^{2}$ is estimated to be smaller than the spatial variance $\sigma_{w}^{2}$. The spatial decay parameter is estimated to be 0.0018 which corresponds to an effective spatial range ([54]) of 1666.7 kilometers that is about half of the maximum distance between any two locations inside the study region. The estimates of $\sigma_{l}^{2}$ for $l=1, \ldots, r$ range from 0.457 to 3.828 and are omitted for brevity.

We now turn to the validation of the ozone summaries: the annual 4th highest maximum and the 3 -year rolling average of these. Table 6 provides the validation statistics. We also report the values of the validation statistics for these summaries obtained using simple kriging using the fields package [35]. In this kriging method, the daily ozone levels are first kriged and then those are aggregated up to the annual levels to mimic our model based approach where we spatially predict on each day and then aggregate the predictions up to the annual levels. As expected, the proposed method is able to perform better in out of sample predictions than standard kriging which is well known to be difficult to beat using model based approaches ([33]). This shows that the model is very accurate in predicting the primary ozone standard based on the annual summaries. Figure 6 examines this in more detail where the predicted values of these summaries are plotted against the observed values. The plot provides evidence of accurate prediction with a slight tendency to over-predict. The actual over prediction percentage for the annual $4 t h$ highest maximum is $52 \%$ while the 
same for the 3 -year rolling averages is slightly higher at $53 \%$ which are reasonable. Hence we proceed to make predictive inference for the ozone standard based on these model based annual summaries.

We perform predictions at 936 locations inside the land-boundary of the eastern US obtained from a regular grid. At each of these sites we spatially interpolate the daily maximum 8-hour average ozone level on each of 153 days in every year using the details in Section 3.3.2. These daily levels are then aggregated up to the annual levels. Figure 7 provides the model based interpolated maps of annual $4 t h$ highest maximum ozone levels for the years 1997-2006. Observed values of these annual maxima from a selected number of sites (data from all the 691 sites are not plotted to avoid clutter) are also superimposed and those show reasonably good agreement with the predicted values. Similarly, Figure 8 plots the model based interpolated maps of the 3 -year rolling averages of the annual 4th highest maximum ozone concentration levels for the years 1999-2006. The 3-year averages increase slightly or remain generally at a constant level from 1999 to 2003 and then decrease. The superimposed observed values of these rolling averages are also in good agreement with the predicted values. The uncertainty maps corresponding to the prediction maps in Figures 7 and 8 showed larger uncertainty for the locations which are farther away from the monitoring sites and are omitted for brevity. The predicted maps show a gradually decreasing trend on ozone levels that can be attributed to gradual reductions in ozone precursor emissions, specially in nitrogen oxides and volatile organic compounds - the main ingredients of ozone. We study the meteorologically adjusted relative percentage trends (see Section 3.3.3) in Figure 9 for 2006 relative to 1997. We observe most of these trends to be negative except for a few sites.

The model based predictive maps of the probability that the 3-year rolling average of the annual 4th highest maximum ozone level is greater than $85 \mathrm{ppb}$, i.e. noncompliance with respect to the primary ozone standard, are provided in Figure 10. The plots show that many areas were out of compliance in the earlier years e.g. in 1999-2003. However, starting in 2004 most areas started to comply with the primary ozone standard and except for few areas shown in the maps. As mentioned above this can be attributed to emission reduction policies adopted be many federal and local government agencies. Another noticeable feature of the Figures 7 and 10 is that the spatial patterns in the successive years maps change relatively slowly. This is also expected as the annual 4th highest ozone levels and the 3-year rolling averages of the annual $4 t h$ highest ozone levels change very gradually, see Figure 3 where the observed values of these have been plotted. 


\section{Relevance to business and industry}

The hierarchical modeling methods developed in this paper allow accurate modeling of localized spatial variation that may change over time. This in turn enables accurate spatial prediction and inference at the highest spatial resolution at the point-level. The general spatio-temporal modeling methods are widely applicable in any suitable industrial example settings. For example, consider the industrial corrosion problem where it is important to know the extent of corrosion at any particular location as discussed in [46]. Corrosion is a common industrial problem that affects furnaces, pipelines, storage tanks, valves, nozzles and many other systems in the oil and other industries. The proposed dynamic space-time models enable early identification of corrosion that may result in the initiation of preventative measures and accurate forecasting of such events in the future. A concrete modeling example is outlined below.

Consider the modeling of corrosion in a large industrial furnace as described in [46]. Health and safety concerns require inspection of the furnace at regular time intervals and recording of the vessel wall thickness at a large number of sites in the furnace. According to past experience of the experts, it is reasonable to assume that the wall thickness will, on average, deteriorate continuously if operating conditions remain constant, and that current corrosion rate is the best estimator of the future corrosion rate. Therefore, the wall thickness is modeled with a locally linear trend as in (4) with details modified as follows.

Let $Z(\mathbf{s}, t)$ denote the observed wall thickness value at a location $\mathbf{s}$ and time $t$. We drop the subscript $l$ since time here can be described by just one index $t$. We also suppose that there are observed covariates, denoted by $\mathbf{x}_{l}(\mathbf{s}, t)$, that may help explain corrosion in the wall thickness. For example, components of $\mathbf{x}_{l}(\mathbf{s}, t)$ may record the furnace usage history in the period before time $t$, and/or an exposure characteristics at location s at time $t$ such as temperature. As in previous section we introduce two sets of independent error distributions: one spatial, denoted by $\eta(\mathbf{s}, t)$, and the other a measurement error, $\epsilon(\mathbf{s}, t)$. As in (4) we can now write the model as:

$$
Z(\mathbf{s}, t)=\mathbf{x}^{\prime}(\mathbf{s}, t) \boldsymbol{\beta}(\mathbf{s})+\eta(\mathbf{s}, t)+\epsilon(\mathbf{s}, t) .
$$

In this model $\boldsymbol{\beta}(\mathbf{s})$ are unknown spatially varying regression co-efficients that can be estimated from the data. If, however, there are no measurable covariates present then we can simply assume the time invariant intercept process $\beta_{0}(\mathbf{s})$ that signifies the overall thickness that will remain even after the end of the life of the vessel. A zero mean Gaussian process prior, see for example, [54] can be assumed for the $\beta_{0}(\mathbf{s})$ process.

A suitable dynamic model must be assumed for the spatially correlated error process $\eta(\mathbf{s}, t)$. Following the development in this paper we assume an auto-regressive model as given in (5). This model for the spatial residuals is intuitively sensible 
here because of the above assumption that the wall thickness deteriorates on average under constant operating conditions. This also justifies the auto-regressive models proposed here instead of the random-walk models, see for example, [54]. The drift term, $\mathbf{x}^{\prime}(\mathbf{s}, t) \boldsymbol{\beta}(\mathbf{s})$, in the overall model can be used to accommodate any effects of change in these conditions. The remaining model and prior distributions are assumed to be the same as in Section 3. The problem of handling large spatial and spatiotemporal industrial data, such as the wall thickness data, can be alleviated by the GPP based modeling developments of this paper. Many specific implementation parameters such as the knot size need to be chosen from a suitable sensitivity study.

The Bayesian spatio-temporal models can potentially solve a very important practical forecasting problem as follows. Often, it is of interest to assess the probability that the wall thickness, $Z\left(\mathbf{s}^{\prime}, t\right)$, at any location $\mathbf{s}^{\prime}$ at any future time point $t$ is less than a given threshold design value. These types of failure probabilities can be easily estimated by the methods developed in Sections 3.3.2 and 3.3.4. Moreover, these probabilities can be plotted on a map that can be continuously updated once new data become available.

The model validation methods by using hold-out data provide automatic ways of checking the full model and its assumptions. These out of sample validation statistics can also be used to compare all the competing modeling strategies discussed in this paper since the one of the main objectives of the modeling exercise here is to obtain out of sample predictions and forecasts.

The discussion here points to the possibility that future investigations with real data sets are likely to lead to fruitful results especially relevant to businesses and industries. However, as is well known, the take-up of the stochastic models by the businesses often does not proceed at a desirable fast pace due to the lack of availability of suitable software packages that implement the methods. This is where the $\mathrm{R}$ package spTimer that has been developed alongside this paper and is freely available can help. This package can do the model fitting, prediction and forecasting for large space-time data sets.

Lastly, we note that the substantive environmental modeling example of this paper is also of direct relevance to many businesses and industries. For example, the accurate spatial prediction of the long term trends in air pollution is likely to influence current and future emission reduction policies. These policies will directly affect the method and the cost of industrial production which will have long-term repercussions in the local economy. Further general discussions of the developed methods are provided in the next section. 


\section{Discussion}

A fast hierarchical Bayesian auto-regressive model for both spatially and temporally rich data sets has been developed in this paper. The methods have been shown to be accurate and feasible for simultaneous modelling and analysis of a large data set with more than a million observations using computationally intensive MCMC sampling algorithms. The proposed auto-regressive models have been shown to validate better than standard Kriging for out of sample predictions.

Specifically, the methods have been illustrated for evaluating meteorologically adjusted trends in the primary ozone standard in the eastern US over a 10 year period from 1997-2006. To our knowledge no such Bayesian model based analysis exists for the same data and the same modelling purposes. An important utility of the high resolution space-time model lies in the ability to predict the primary ozone standard at any given location for the modelled period. This helps in understanding spatial patterns and trends in ozone levels both at the meteorologically adjusted and unadjusted levels which in turn will help in evaluating emission reduction policies that directly affect many industries.

The proposed methods can also be used in conjunction with a spatio-temporal downscaler model for incorporating output from numerical models as discussed in [47] and [48]. The essence of the down-scaler model is to use the grid-level output from numerical models as a covariate in the point level model such as (4). This type of space and time varying covariate information enriches the regression settings like the one in the ARGPP model (8) and in fact will allow us to estimate spatially and/or temporally varying regression coefficients $([49]$ and [47]) in the model, see also [50]. A downscaler model with or without our ARGPP approach, however, cannot be used for assessing meteorologically adjusted trends since the outputs of the numerical models are based on the meteorological data and including the meteorological data again in the model will lead to multi-collinearity problems.

An $\mathrm{R}$ package, spTimer, has been developed for implementing the models for analysing large volumes of space-time data and will be made publicly available from the $\mathrm{R}$ archive network CRAN (http://cran.r-project.org/). The spTimer package with its ability to fit, predict and forecast using a number of space-time models can be used for modelling a wide variety of large space-time data.

Multivariate space-time modelling for large data sets is a challenging task. The methods developed in this paper can be extended to cope with a multivariate spacetime random effect that can be specified using a linear model of coregionalisation, see e.g., [51]. An alternative of this last method is to specify the multivariate response conditionally, see e.g., [52] where ozone concentration levels and particulate matter data have been modelled jointly. 


\section{Appendix}

The Gibbs sampler is implemented by repeatedly simulating the missing observations and all the unknown model parameters from their full conditional distributions. The joint posterior distribution (10) is used to derive the full conditional distributions listed below.

- Any missing observation $Z_{l}^{*}\left(\mathbf{s}_{i}, t\right)$ has the full conditional distribution $N\left(\mathbf{x}_{l}^{\prime}\left(\mathbf{s}_{i}, t\right) \boldsymbol{\beta}+\tilde{\eta}_{l}\left(\mathbf{s}_{i}, t\right), \sigma_{\epsilon}^{2}\right)$.

- The full conditional distribution of $\boldsymbol{\beta}$ is $N(\Delta \chi, \Delta)$ where,

$$
\begin{gathered}
\Delta^{-1}=\frac{1}{\sigma_{\epsilon}^{2}} \sum_{l=1}^{r} \sum_{t=1}^{T} X_{l t}^{\prime} X_{l t}+10^{-4} I, \\
\chi=\frac{1}{\sigma_{\epsilon}^{2}} \sum_{l=1}^{r} \sum_{t=1}^{T} X_{l t}^{\prime}\left(\mathbf{Z}_{l t}-A \mathbf{w}_{l t}\right) .
\end{gathered}
$$

- The full conditional distribution of $\rho$ is $N(\Delta \chi, \Delta) I(0<\rho<1)$ where,

$$
\begin{gathered}
\Delta^{-1}=\sum_{l=1}^{r} \sum_{t=1}^{T} \mathbf{w}_{l t-1}^{\prime} Q_{w} \mathbf{w}_{l t-1}+10^{-4} \\
\chi=\sum_{l=1}^{r} \sum_{t=1}^{T} \mathbf{w}_{l t-1}^{\prime} Q_{w} \mathbf{w}_{l t}
\end{gathered}
$$

where $Q_{w}=\Sigma_{w}^{-1}$.

- The full conditional distribution of $\frac{1}{\sigma_{\epsilon}^{2}}$ is given by:

$$
G\left(\frac{N}{2}+a, b+\frac{1}{2} \sum_{l=1}^{r} \sum_{t=1}^{T}\left(\mathbf{Z}_{l t}-X_{l t} \boldsymbol{\beta}-A \mathbf{w}_{l t}\right)^{\prime}\left(\mathbf{Z}_{l t}-X_{l t} \boldsymbol{\beta}-A \mathbf{w}_{l t}\right)\right)
$$

- Similarly, the full conditional distribution of $\frac{1}{\sigma_{w}^{2}}$ is written as:

$$
G\left(\frac{m r T}{2}+a, b+\frac{1}{2} \sum_{l=1}^{r} \sum_{t=1}^{T}\left(\mathbf{w}_{l t}-\rho \mathbf{w}_{l t-1}\right)^{\prime} Q_{w}\left(\mathbf{w}_{l t}-\rho \mathbf{w}_{l t-1}\right)\right)
$$

- The full conditional distribution of $\mathbf{w}_{l t}$ is given by: $N(\Delta \chi, \Delta)$ where

$$
\Delta^{-1}=\frac{1}{\sigma_{\epsilon}^{2}} A^{\prime} A+Q_{w}+\rho^{2} Q_{w}
$$




$$
\chi=\frac{1}{\sigma_{\epsilon}^{2}} A^{\prime}\left(\mathbf{Z}_{l t}-X_{l t} \boldsymbol{\beta}\right)+Q_{w} \mathbf{w}_{l t-1}+Q_{w} \mathbf{w}_{l t+1},
$$

for $1 \leq t<T$. For $t=T$, we have

$$
\begin{gathered}
\Delta^{-1}=\frac{1}{\sigma_{\epsilon}^{2}} A^{\prime} A+Q_{w} \\
\chi=\frac{1}{\sigma_{\epsilon}^{2}} A^{\prime}\left(\mathbf{Z}_{l t}-X_{l t} \boldsymbol{\beta}\right)+Q_{w} \mathbf{w}_{l t-1} .
\end{gathered}
$$

- The full conditional distribution of $\mathbf{w}_{l 0}$ is given by $N(\Delta \chi, \Delta)$ where,

$$
\begin{gathered}
\Delta^{-1}=\rho^{2} Q_{w}+Q_{0}^{-1} \\
\chi=\rho Q_{w} \mathbf{w}_{l 1}+\mu_{l} \Sigma_{0}^{-1} \mathbf{1}_{m},
\end{gathered}
$$

where $Q_{0}=\Sigma_{0}^{-1}$.

- The full conditional distribution of $\sigma_{l}^{2}$ for $l=1, \ldots, r$ is given by:

$$
G\left(\frac{m}{2}+a, b+\frac{1}{2} \mathbf{w}_{l 0} \mathbf{S}_{0}^{-1} \mathbf{w}_{l 0}\right) .
$$

- The full conditional distribution of $\phi$ is not available in closed form. The log of the conditional posterior density (upto an additive constant) is given by:

$$
\log \pi(\phi \mid \ldots)=\log \pi\left(\phi_{w}\right)-\frac{r T}{2} \log \left|S_{w}\right|-\frac{1}{2} \sum_{l=1}^{r} \sum_{t=1}^{T}\left(\mathbf{w}_{l t}-\rho \mathbf{w}_{l t-1}\right)^{\prime} Q_{w}\left(\mathbf{w}_{l t}-\rho \mathbf{w}_{l t-1}\right)
$$

Similarly, the log of the conditional posterior density of $\phi_{0}$ (upto an additive constant) is given by:

$$
\log \pi\left(\phi_{0} \mid .\right)=\log \pi\left(\phi_{0}\right)-\frac{r}{2} \log \left(\left|S_{0}\right|\right)-\frac{1}{2} \sum_{l=1}^{r} \frac{1}{\sigma_{l}^{2}} \mathbf{w}_{l 0} \mathbf{S}_{0}^{-1} \mathbf{w}_{l 0} .
$$

When the prior distributions $\pi(\phi)$ and $\pi\left(\phi_{0}\right)$ are assumed to be discrete that the above conditional posterior distributions are also discrete having the same mass points as those of the prior distributions. However, when the prior distributions are continuous we use a Metropolis-Hastings random walk algorithm to sample from these conditional distributions. This algorithm is tuned to have an optimal acceptance rate between $20 \%$ to $40 \%$, see e.g., [53]. The algorithm is implemented on the log-scale for $\phi$, see [54] for further details.

\section{Acknowledgements}

We thank Professor Alan Gelfand and Dr David Holland for many helpful comments and suggestions. 


\section{References}

[1] Banerjee, S., Gelfand, A.E., Finley, A.O. \& Sang, H. (2008). Gaussian Predictive Process Models for Large Spatial Data Sets. Journal of the Royal Statistical Society, Series: B, 70, 825-848.

[2] Franklin, C. (1992). An introduction to geographic information systems: linking maps to databases. Database, 15, 13-21.

[3] Cressie, N. and Wikle, C. K. (2011) Statistics for Spatio-Temporal Data. John Wiley \& Sons.

[4] Sahu, S. K., Gelfand, A. E. and Holland, D. M. (2007). High Resolution SpaceTime Ozone Modeling for Assessing Trends. Journal of the American Statistical Association, 102, 1221-1234.

[5] Cameletti, M., Ignaccolo, R. and Bande, S. (2009). Comparing Air Quality Statistical Models. Technical Report. University of Bergamo, Italy.

[6] Sahu, S. K. and Bakar, K. S. (2011) A comparison of Bayesian Models for Daily Ozone Concentration Levels Statistical Methodology, DOI: 10.1016/j.stamet.2011.04.009.

[7] Banerjee, S., Carlin, B.P. and Gelfand, A.E. (2004). Hierarchical Modeling and Analysis for Spatial Data. Chapman and Hall/CRC.

[8] Cressie, N.A.C (1993). Statistics for Spatial Data. John Wiley and Sons, New York, revised edition.

[9] Haas, T.C. (1995). Local prediction of a spatio-temporal process with an application to wet sulfate deposition. Journal of the American Statistical Association, 90, 1189-1199.

[10] Kammann, E.E. \& Wand, M.P. (2003). Geoadditive models. Applied Statistics, 52, $1-18$.

[11] Xia, G. and Gelfand, A.E. (2006). Stationary Process Approximation for the Analysis of Large Spatial Datasets. Technical Report. Institute of Statistical and Decision Sciences, Duke University, Durham, USA.

[12] Nychka, D. (2000). Spatial-process estimates as smoothers. In Smoothing and Regression: Approaches, Computation, and Application. Eds. M.G. Schimek, 393-424, New York, Wiley.

[13] Stein, M.L. (1999). Statistical Interpolation of Spatial Data: Some Theory for Kriging. New York: Springer. 
[14] Paciorek, C.J. (2007). Computational techniques for spatial logistic regression with large datasets. Computational Statistics and Data Analysis, 51, 3631-3653.

[15] Hartman, L. and Hössjer, O. (2008) Fast kriging of large data sets with Gaussian Markov random fields. Computational Statistics \& Data Analysis, 2331-2349.

[16] Rue, H. and Held, L. (2006). Gaussian Markov Random Fields: Theory and Applications. Boca Raton: Chapman and Hall/CRC.

[17] Reich, B.J., Fuentes, M. and Dunson, D.B. (2011). Bayesian Spatial Quantile Regression. Journal of the American Statistical Association, 106, DOI: 10.1198/jasa.2010.ap09237.

[18] Huang, H.C., Cressie, N. and Gabrosek, J. (2002). Fast, resolution-consistent spatial prediction of global processes from satellite data. Journal of Computational and Graphical Statistics, 11, 63-88.

[19] Johannesson, G. and Cressie, N. (2004). Finding large-scale spatial trends in massive, global, environmental datasets. Environmetrics, 15, 1-44.

[20] Johannesson, G., Cressie, N. and Huang, H.C. (2007). Dynamic multi-resolution spatial models. Environmental and Ecological Statistics, 14, 5-25.

[21] Stein, M.L. (2007). Spatial variation of total column ozone on a global scale. Annals of Applied Statistics, 1, 191-210.

[22] Stein, M.L. (2008). A modelling approach for large spatial datasets. Journal of the Korian Statistical Society, 37, 3-10.

[23] Cressie, N.A.C \& Johannesson, G. (2008). Fixed rank kriging for very large spatial data sets. Journal of the Royal Statistical Society, Series: B, 70, 209226.

[24] Cressie, N., Shi, T., Kang, E. L. (2010). Fixed Rank Filtering for SpatioTemporal Data. Journal of Computational and Graphical Statistics, 19, 724745.

[25] Carroll, R. J., Chen, R., George, E. I., Li, T.H., Newton, H.J., Schmiediche, H. and Wang, N. (1997). Ozone exposure and population density in Harris County, Texas. Journal of the American Statistical Association, 92, 392-404.

[26] Guttorp, P., Meiring, W. and Sampson, P. D. (1994). A Space-time Analysis of Ground-level Ozone Data. Environmetrics, 5, 241-254.

[27] Huang, L. S. and Smith, R. L. (1999). Meteorologically-dependent trends in urban ozone. Environmetrics, 10, 103-118. 
[28] Thompson, M. L., Reynolds, J., Cox, L. H., Guttorp, P., and Sampson, P. D. (2001). A review of statistical methods for the meteorological adjustment of tropospheric ozone. Atmospheric Environment, 35, 617-630.

[29] Huerta, G., Sanso, B., and Stroud, J. R. (2004). A spatiotemporal model for Mexico City ozone levels. Journal of the Royal Statistical Society, Series C, 53, 231-248.

[30] Gilleland, E. and Nychka, D. (2005). Statistical Models for Monitoring and Regulating Ground Level Ozone. Environmetrics, 16, 535-546.

[31] McMillan, N., Bortnick, S. M., Irwin, M. E. and Berliner, M. (2005). A hierarchical Bayesian model to estimate and forecast ozone through space and time. Atmospheric Environment, 39, 1373-1382.

[32] Cocchi, D., Fabrizi, E., and Trivisano, C. (2005). A stratified model for the assessment of meteorologically adjusted trends of surface ozone. Environmental and Ecological Statistics 12, 1195-1208.

[33] Liu, Z., Le, N. D., Zidek, J. V. (2011) An empirical assessment of Bayesian melding for mapping ozone pollution. Environmetrics, 22, 340-353.

[34] McMillan, N., Holland, D. M., and Morara, M., and Feng, J. (2010). Combining numerical model output and particulate data using Bayesian space-time modeling. Environmetrics, 21, 48-65.

[35] Fields Development Team (2006). fields: Tools for Spatial Data. National Center for Atmospheric Research, Boulder, CO. http://www.cgd.ucar.edu/Software/Fields.

[36] Bakar, K. S. (2011). Bayesian Analysis of Daily Maximum Ozone Levels. PhD Thesis, University of Southampton. UK.

[37] Christos, P. (2011). Bayesian Spatial-Temporal Modelling of Air Pollution. PhD Thesis. Bath University, UK.

[38] Bakar, K. S., Awang, N. and Sahu, S. K. (2011) Forecasting next day ozone levels using Gaussian Predictive Processes. Under Preparation.

[39] Finley, A.O., S. Banerjee, and B.P. Carlin. (2007). spBayes: A program for multivariate point-referenced spatial modeling. Journal of Statistical Software, 19:4.

[40] Bakar, K. S. and Sahu, S. K. (2011) spTimer: Spatio-Temporal Bayesian Modelling using R. Under Preparation. 
[41] Gelfand, A. E. and Ghosh, S. K. (1998). Model Choice: A Minimum Posterior Predictive Loss Approach. Biometrika, 85, 1-11.

[42] Gelman, A. and Rubin, D.B. (1992). Inference from iterative simulation using multiple sequences (with discussion). Statistical Science, 7, 457-511.

[43] Inconsistent estimation and asymptotically equal interpolations in model-based geostatistics. Journal of the American Statistical Association, 99, 250-261.

[44] Sahu, S. K., Gelfand, A. E. and Holland, D. M. (2010). Fusing point and areal level space-time data with application to wet deposition. Journal of the Royal Statistical Society, C, 59, 77-103.

[45] Finley, A.O., Sang, H., Banerjee, S., and Gelfand, A. (2009). Improving the performance of predictive process modeling for large datasets. Computational Statistics and Data Anlaysis, 53, 2873-2884.

[46] Little, J., Goldstein, M., Jonathan, P. and den Heijer K. (2004). Spatiotemporal modelling of corrosion in an industrial furnace. Appl. Stochastic Models Bus. Ind., 20, 219238.

[47] Berrocal, V. J., Gelfand, A. E. and Holland, D. M. (2010). A Spatio-Temporal Downscaler for Output From Numerical Models. Journal of the Agricultural, Biological and Environmental Statistics, 15, 176-197.

[48] Sahu, S. K., Yip, S. and Holland, D. M. (2009). Improved space-time forecasting of next day ozone concentrations in the eastern US. Atmospheric Environment, doi:10.1016/j.atmosenv.2008.10.028.

[49] Gelfand, A. E., Kim, H. J., Sirmans, C. F., and Banerjee, S. (2003). Spatial modeling with spatially varying coefficient processes. Journal of the American Statistical Association, 98, 387-396.

[50] Nobre, A. A., Sanso, B., and Schmidt, A. M. (2011) Spatially Varying Autoregressive Processes. Technometrics, 53, 310-321.

[51] Gelfand, A. E. Schmidt, A. M. Banerjee, S. and Sirmans, C. F. (2004). Nonstationary Multivariate Process Modelling through Spatially Varying Coregionalization (with discussion). Test, 2, 1-50.

[52] Daniels, M.J., Zhou, Z. G., and Zou, H. (2006) Conditionally specified spacetime models for multivariate processes. Journal of Computational and Graphical Statistics, 15, 157-177. 
[53] Gelman, A., Roberts, G. O., and Gilks, W. R. (1996). Efficient Metropolis Jumping Rules. In Bayesian Statistics 5, edited by J. M. Bernardo, J. O. Berger, A. P. Dawid and A. F. M. Smith. Oxford University Press, pp 599-607.

[54] Sahu, S. K. (2011). Hierarchical Bayesian models for space-time air pollution data In Handbook of Statistics-Vol 30. Time Series Analysis, Methods and Applications. Editors: T Subba Rao and C R Rao. Elsevier Publishers, Holland. To appear.

\begin{tabular}{r|ccccc}
\hline Knot size & 26 & 40 & 60 & 107 & 138 \\
\hline RMSE & 6.31 & 6.19 & 6.17 & 6.07 & 6.06 \\
MAE & 4.58 & 4.48 & 4.44 & 4.37 & 4.36 \\
\hline
\end{tabular}

Table 1: Values of the two validation criteria for different knot sizes for the four states example.

\begin{tabular}{rrrrr}
\hline Parameter & Mean & SD & $2.5 \%$ & $97.5 \%$ \\
\hline & \multicolumn{4}{c}{ ARHIER } \\
\hline Intercept & 4.447 & 0.061 & 4.346 & 4.541 \\
Max.Temp. & 0.016 & 0.001 & 0.013 & 0.018 \\
RH & -0.314 & 0.010 & -0.325 & -0.302 \\
WDSP & -0.061 & 0.002 & -0.065 & -0.057 \\
$\rho$ & 0.523 & 0.002 & 0.519 & 0.526 \\
$\sigma_{\epsilon}^{2}$ & 0.056 & 0.001 & 0.055 & 0.058 \\
$\sigma_{\eta}^{2}$ & 0.537 & 0.038 & 0.527 & 0.540 \\
$\phi$ & 0.0120 & 0.0006 & 0.0119 & 0.0121 \\
\hline \multicolumn{5}{c}{ ARGPP } \\
\hline Intercept & 6.353 & 0.056 & 6.224 & 6.445 \\
MaxTemp & 0.060 & 0.001 & 0.057 & 0.063 \\
RH & -0.179 & 0.009 & -0.198 & -0.160 \\
WDSP & -0.033 & 0.001 & -0.036 & -0.031 \\
$\rho$ & 0.102 & 0.003 & 0.095 & 0.109 \\
$\sigma_{\epsilon}^{2}$ & 0.169 & 0.001 & 0.167 & 0.171 \\
$\sigma_{w}^{2}$ & 0.457 & 0.004 & 0.449 & 0.466 \\
$\phi$ & 0.0036 & 0.0001 & 0.0030 & 0.0041 \\
\hline
\end{tabular}

Table 2: Parameter estimates of the two AR models. 


\begin{tabular}{rrrrrrr}
\hline & $\mathrm{P}$ & $\mathrm{G}$ & $\mathrm{P}+\mathrm{G}$ & RMSE & MAE & Coverage (\%) \\
\hline Full & $90,807.32$ & $41,077.80$ & $131,885.10$ & 6.82 & 5.04 & 93.50 \\
GPP & $40,893.51$ & $28,143.46$ & $69,036.97$ & 6.07 & 4.37 & 92.88 \\
\hline
\end{tabular}

Table 3: Model comparison results for the ARHIER and ARGPP models.

\begin{tabular}{ccccc}
\hline \multicolumn{5}{c}{ Knot Sizes } \\
\hline & 269 & 156 & 105 & 68 \\
\hline RMSE & 6.41 & 6.42 & 6.78 & 7.09 \\
MAE & 4.73 & 4.75 & 5.02 & 5.26 \\
\hline
\end{tabular}

Table 4: Two model validation criteria for different knot sizes

\begin{tabular}{rrrrr}
\hline & Mean & sd & $2.5 \%$ & $97.5 \%$ \\
\hline Intercept & 6.817 & 0.101 & 6.604 & 6.991 \\
MaxTemp & 0.027 & 0.001 & 0.025 & 0.029 \\
$\mathrm{RH}$ & -0.243 & 0.004 & -0.251 & -0.234 \\
WDSP & -0.009 & 0.002 & -0.013 & -0.006 \\
$\rho$ & 0.132 & 0.002 & 0.128 & 0.136 \\
$\sigma_{\epsilon}^{2}$ & 0.266 & 0.001 & 0.265 & 0.267 \\
$\sigma_{w}^{2}$ & 0.729 & 0.014 & 0.708 & 0.770 \\
$\phi$ & 0.0018 & 0.0001 & 0.0017 & 0.0019 \\
\hline
\end{tabular}

Table 5: Parameter estimates of the fitted GPP based AR model for the eastern US data.

\begin{tabular}{ccccc}
\hline \multicolumn{3}{c}{ Annual 4 th highest } & \multicolumn{2}{c}{ 3-year average } \\
\hline & Kriging & Model & Kriging & Model \\
RMSE & 5.41 & 5.24 & 4.27 & 4.21 \\
MAE & 4.38 & 4.17 & 3.51 & 3.36 \\
\hline
\end{tabular}

Table 6: Two validation criteria for the annual ozone summaries 


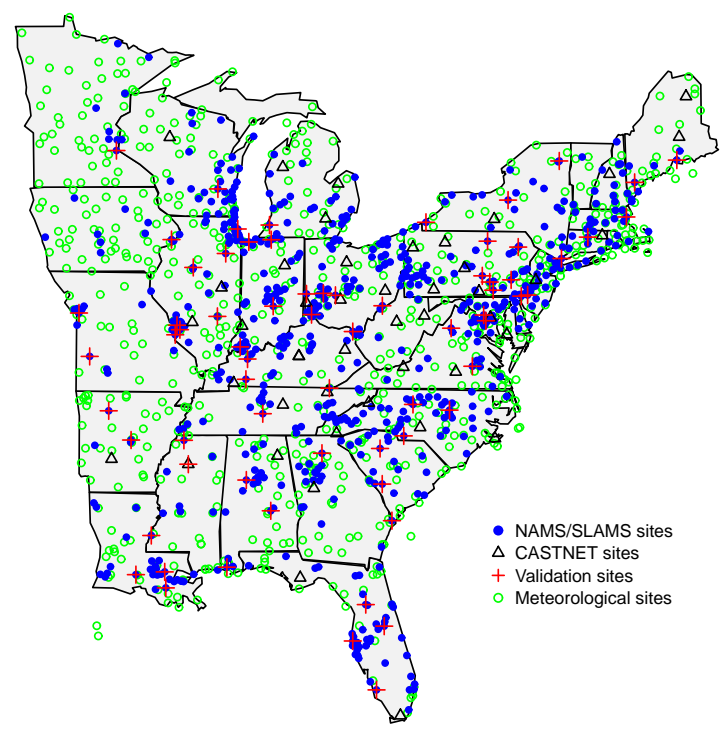

Figure 1: A map showing the locations of the ozone concentration and weather monitoring sites.

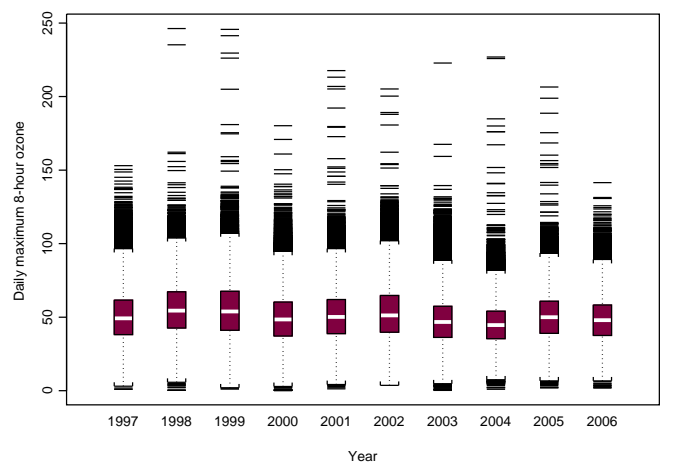

Figure 2: Boxplot of daily maximum eight-hour ozone concentration levels by years. 

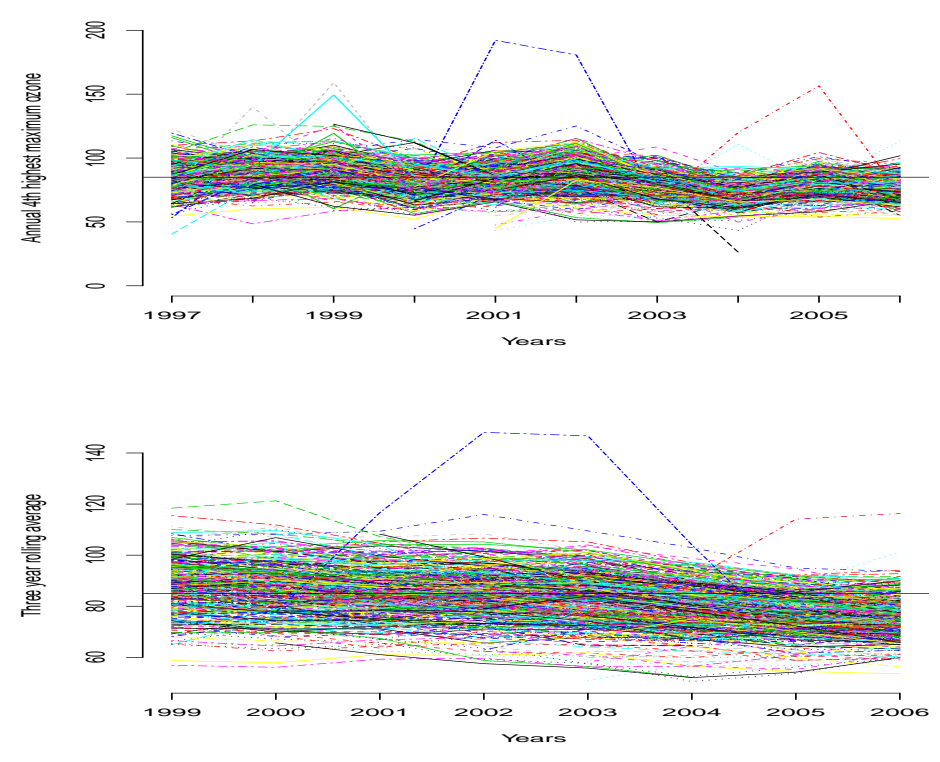

Figure 3: Time series plots of the ozone concentration summaries from 691 sites: (a) annual 4 th highest maximum and (b) 3-year rolling average of the annual 4 th highest maximum.

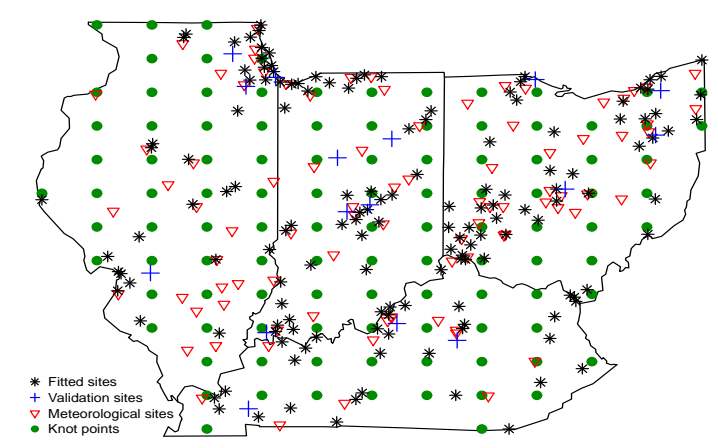

Figure 4: A map of the four states: Illinois, Indiana, Ohio, and Kentucky showing 164 ozone monitoring sites consisting of 148 fitting and 16 validation sites, 88 meteorological sites and 107 knot locations. 


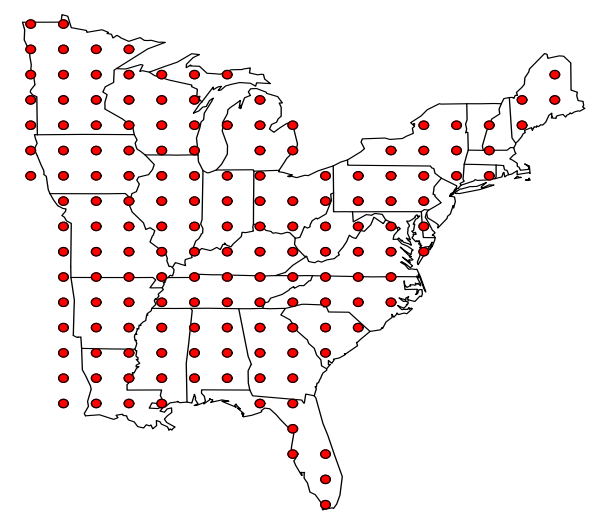

Figure 5: A map of the eastern US with 156 knot points superimposed.

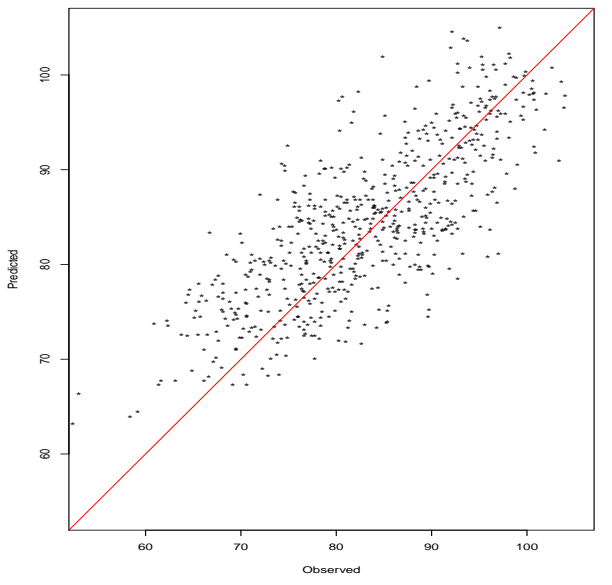

(a)

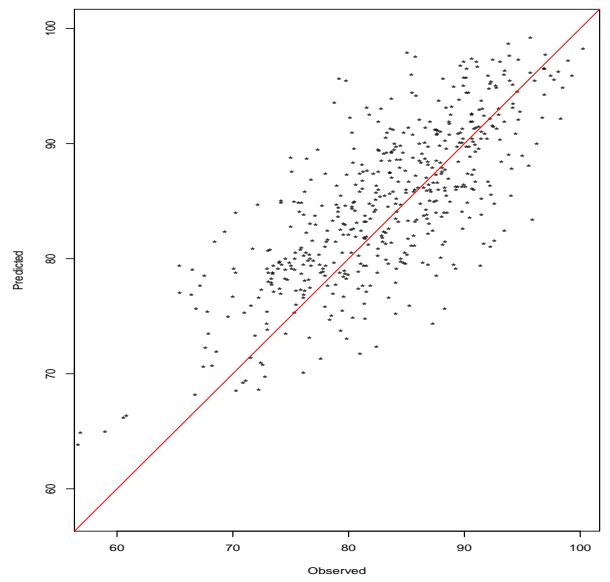

(b)

Figure 6: Scatter plots of the prediction against the observed values, (a): annual 4th highest maximum, (b) 3-year rolling average of the annual 4th highest maximum. The $y=x$ line is superimposed. 


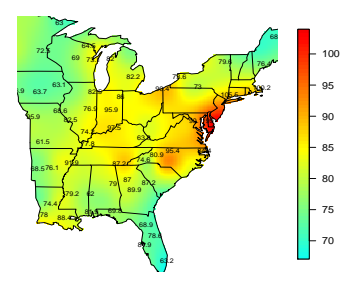

(a)

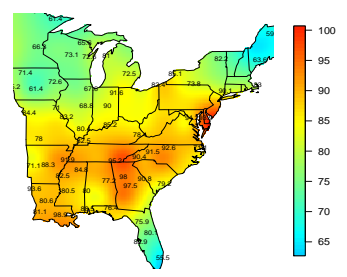

(d)

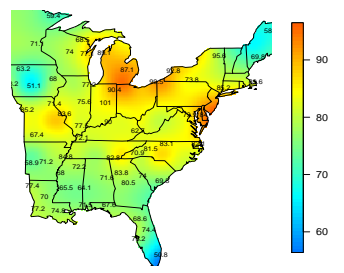

$(\mathrm{g})$

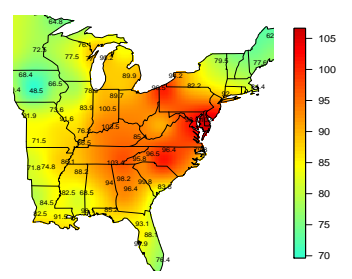

(b)

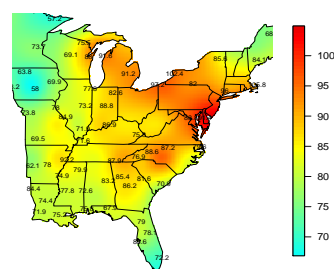

(e)

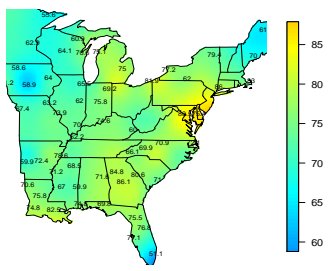

(h)

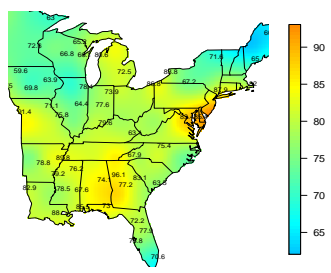

(j)

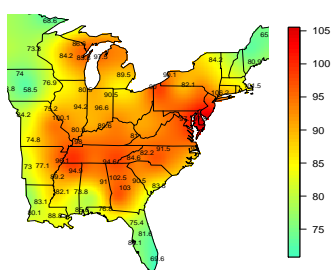

(c)

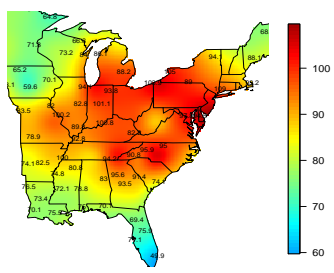

(f)

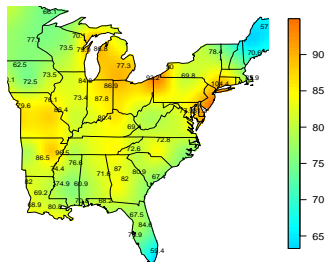

(i)

Figure 7: Model based interpolation of the annual 4th highest maximum ozone levels for 10 years, panel (a) for 1997 to panel (j) for 2006. Observed data from a few selected sites, to enhance readability, are superimposed. 


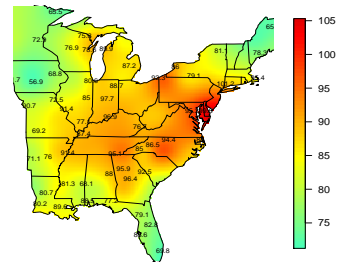

(a)

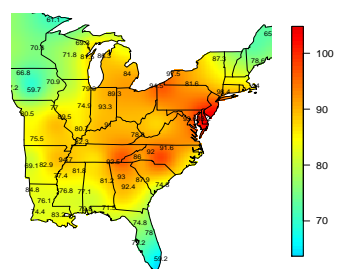

(d)

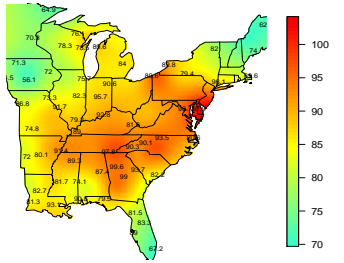

(b)

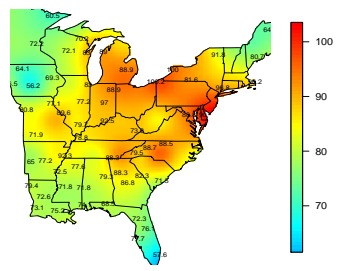

(e)

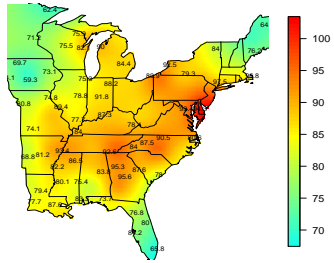

(c)

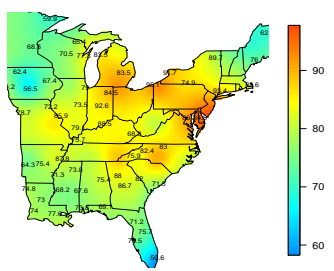

(f)

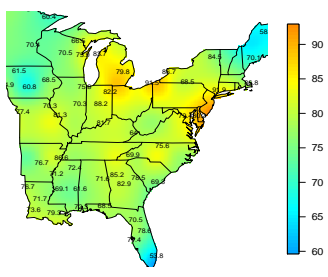

$(\mathrm{g})$

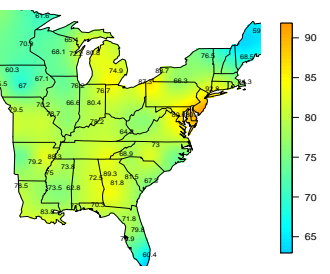

(h)

Figure 8: Model based interpolation of the 3-year rolling average of the annual 4th highest maximum ozone levels for 8 years, panel (a) for 1999 to panel (h) for 2006. Observed data from a few selected sites, to enhance readability, are superimposed. 


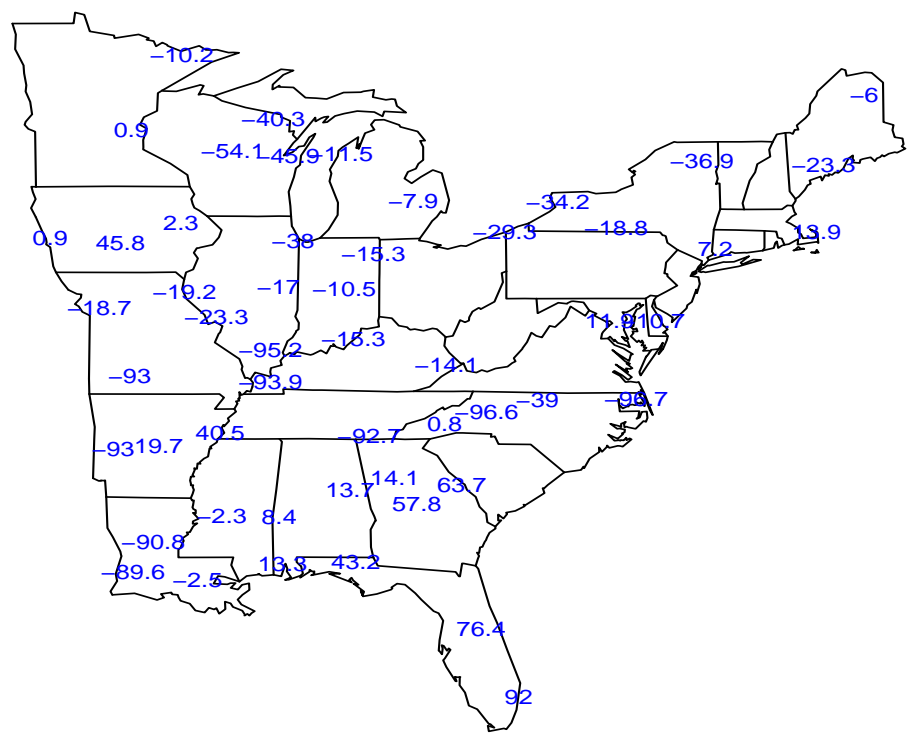

Figure 9: Relative percentage trends for the meteorologically adjusted levels for the year 2006 relative to 1997. 


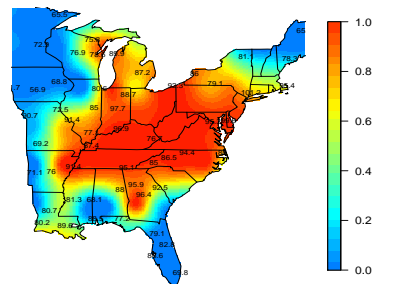

(a)

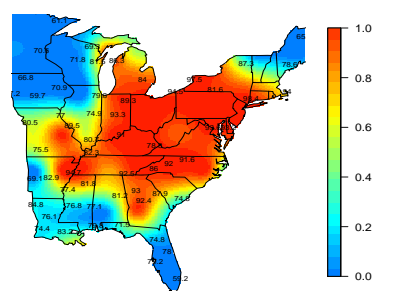

(d)

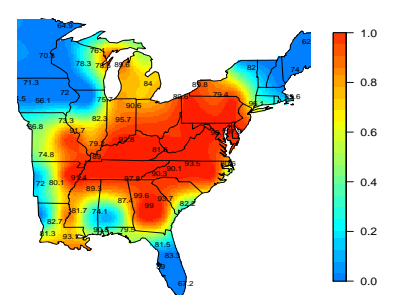

(b)

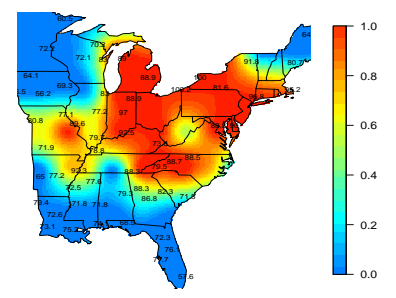

(e)

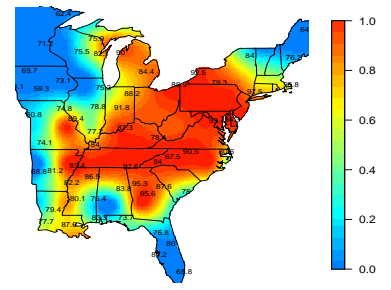

(c)

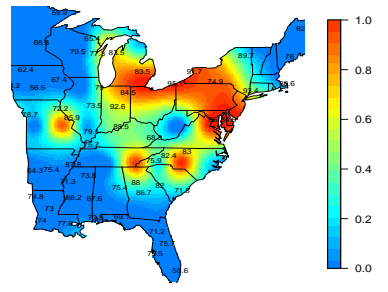

(f)

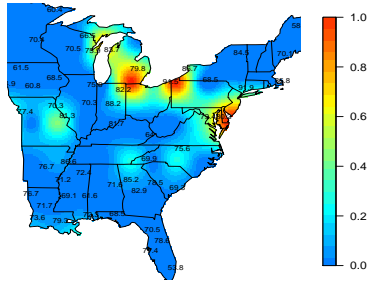

(g)

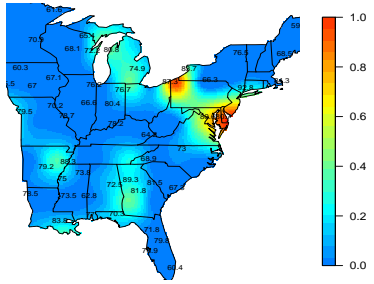

(h)

Figure 10: Model based interpolated maps of the probability that the 3-year rolling average of the annual $4 t h$ highest maximum ozone level is greater than $85 \mathrm{ppb}$ for the years 1999 panel (a) to 2006 panel (h). Observed 3-year averages from a few selected sites, to enhance readability, are superimposed. 